Review Article

\title{
Plasma Circulating Tumor DNA Levels for the Monitoring of Melanoma Patients: Landscape of Available Technologies and Clinical Applications
}

\author{
Benoit Busser, ${ }^{1,2}$ Julien Lupo, ${ }^{3,4}$ Lucie Sancey, ${ }^{2}$ Stéphane Mouret, ${ }^{5}$ \\ Patrice Faure, ${ }^{1,6}$ Joel Plumas, ${ }^{2,7}$ Laurence Chaperot, ${ }^{2,7}$ Marie Thérèse Leccia, ${ }^{2,5}$ \\ Jean Luc Coll, ${ }^{2}$ Amandine Hurbin, ${ }^{2}$ Pierre Hainaut, ${ }^{2}$ and Julie Charles ${ }^{2,5}$ \\ ${ }^{1}$ Biochemistry Pharmacology and Toxicology Department, Grenoble University Hospital, Grenoble, France \\ ${ }^{2}$ Institute for Advanced Biosciences, UGA/INSERM U1209/CNRS UMR5309, Grenoble, France \\ ${ }^{3}$ Virology Laboratory, Grenoble University Hospital, Grenoble, France \\ ${ }^{4}$ Institut de Biologie Structurale, CEA-CNRS UMR 5075/UGA, Grenoble, France \\ ${ }^{5}$ Dermatology Unit, Grenoble University Hospital, Grenoble, France \\ ${ }^{6}$ UGA, Laboratory of Hypoxy Physiopathology Study, INSERM U1042, Grenoble, France \\ ${ }^{7}$ EFS Rhone-Alpes-Auvergne, Grenoble, France
}

Correspondence should be addressed to Benoit Busser; bbusser@chu-grenoble.fr

Received 11 August 2016; Revised 8 March 2017; Accepted 22 March 2017; Published 6 April 2017

Academic Editor: Pascale Quatresooz

Copyright (C) 2017 Benoit Busser et al. This is an open access article distributed under the Creative Commons Attribution License, which permits unrestricted use, distribution, and reproduction in any medium, provided the original work is properly cited.

\begin{abstract}
Melanoma is a cutaneous cancer with an increasing worldwide prevalence and high mortality due to unresectable or metastatic stages. Mutations in BRAF, NRAS, or KIT are present in more than $60 \%$ of melanoma cases, but a useful blood-based biomarker for the clinical monitoring of melanoma patients is still lacking. Thus, the analysis of circulating tumor cells (CTCs) and/or cellfree circulating tumor DNA (ctDNA) analysis from blood (liquid biopsies) appears to be a promising noninvasive, repeatable, and systemic sampling tool for detecting and monitoring melanoma. Here, we review the molecular biology-based strategies used for ctDNA quantification in melanoma patients, as well as their main clinical applications. Droplet digital PCR (ddPCR) and next generation sequencing (NGS) technologies appear to be two versatile and complementary strategies to study rare variant mutations for the detection and monitoring of melanoma progression. Among the different clinical uses of ctDNA, we highlight the assessment of molecular heterogeneity and the identification of genetic determinants for targeted therapy as well as the analysis of acquired resistance. Importantly, ctDNA quantification might also be a novel biomarker with a prognostic value for melanoma patients.
\end{abstract}

\section{Introduction}

It is well established that circulating tumor DNA (ctDNA) is a valid surrogate tumor biomarker for monitoring tumor burden and responses to anticancer therapies $[1,2]$. This minimally invasive method to access cancer-derived DNA is also potentially useful for monitoring solid tumors and would avoid the need to perform repeated invasive biopsy procedures [3].

It was recently shown in a prospective proof-of-concept study that the monitoring of metastatic breast cancer via
ctDNA was a both highly specific and sensitive strategy [4]. These results even suggest that ctDNA has better sensitivity than the well-established breast tumor biomarker (TM) carbohydrate antigen (CA 15-3). Similarly, serum levels of carcinoembryonic antigen (CEA), CA 19-9, and prostate specific antigen (PSA) can be used as surrogate markers of tumor burden changes in colon, pancreas, and prostate carcinomas. However, there is no valid and specific blood biomarker that is currently used either for the assessment of melanoma burden/recurrence or for clinical monitoring of the disease. The only TM accepted as a standard prognostic 
factor of survival for melanoma is lactate dehydrogenase $(\mathrm{LDH})$, which is a nonspecific enzyme that can be elevated in various benign or malignant diseases [5].

A relevant blood biomarker for routine clinical melanoma monitoring is thus highly and urgently needed. Melanomas are among the cancers that harbor the highest number of mutations per tumor [6] and ctDNA is detectable in about $80 \%$ of cases, with more than 1000 mutant fragments per $5 \mathrm{~mL}$ of plasma [7]. Mutations, deletions, or amplifications in $B R A F, N R A S, T P 53$, or KIT are generally present in approximately $85 \%$ of melanomas [8]. These high-frequency genetic alterations can be found in the blood of melanoma patients and make it possible to distinguish ctDNA from circulating normal DNA. They could collectively serve as specific molecular biomarkers to track ctDNA levels and as personalized biomarkers of melanoma disease.

$B R A F$ is the most frequently mutated gene in melanoma [8]. From 43 to $66 \%$ of cutaneous melanomas carry BRAF mutations, among which the BRAF V600E transversion is the most common (80\%), followed by V600K (12\%), V600R (5\%), V600M (4\%), and V600D (<5\%) [9]. These activating $B R A F$ mutations induce the constitutive downstream activation of the MEK-ERK signaling pathway, leading to tumor proliferation and survival [10].

NRAS encodes a small GTPase, which was the first protooncogene discovered in melanoma [11], and is found to be mutated in approximately $20 \%$ of cases [12]. Both BRAF and NRAS mutations are predictors of poorer outcome and lower overall survival (OS) of patients than those with nonmutated melanoma [13].

KIT is a gene which is found mutated in approximately $3 \%$ of melanomas [8]. The positive detection of KIT has also been successfully performed in the peripheral blood of patients with gastrointestinal stromal tumors $[14,15]$. Patients with melanomas harboring KIT mutations are eligible for imatinib therapy [16].

Hence, there is a reason to believe that ctDNA will play the unhoped-for role of the currently missing gold-standard blood-based biomarker for the monitoring of melanoma in the near future.

Here, we review the existing molecular biology approaches that have been used for ctDNA quantification for melanoma patients and describe the main clinical applications and associated results that were obtained.

\section{Technical Strategies for ctDNA Detection and Quantification}

Patients with solid malignancies have higher levels of normal (wild-type) circulating cell-free DNA than healthy individuals [37]. Most conventional PCR-based methods, such as classical Sanger sequencing or pyrosequencing, can detect mutant alleles but are limited by the presence of disproportionate amounts of wild-type alleles in the blood. These two methods can only fulfill the requirements for ctDNA quantification for patients with very high levels of mutant ctDNA, which is rare in plasma [38].

Detecting somatic genetic alterations in the circulation is challenging, but novel approaches have facilitated sensitive and specific detection at low levels. Several recently developed methodologies, such as allele-specific amplification refractory mutation system PCR (ARMS), bead emulsification amplification and magnetics (BEAMing) technology, allele-specific PCR (AS-PCR), droplet digital PCR (ddPCR), and next generation sequencing (NGS), have been used to detect and quantify rare variants in the blood of melanoma patients, with analytical sensitivity ranging from 0.005 to $5 \%$ (Table 1).

One of the first studies dedicated to the evaluation of both an innovative ctDNA quantification method and its clinical utility in melanoma showed that quantitative realtime clamp reverse transcription PCR by peptide locked nucleic acid (PNA) and locked nucleic acid hybrid probes (LNAs) detected serum BRAF V600E with a sensitivity of $0.001 \%$. The use of allele-specific blockers to suppress the amplification of the wild-type allele was also chosen for the screening of BRAF mutant alleles in plasma via competitive allele-specific PCR (CastPCR) [25]. However, the analytical sensitivity was lower at $0.5 \%$.

The detection of $B R A F$ mutations in isolated CTCs from $B R A F$-mutated melanoma patients has been performed after an obligatory step of whole genome amplification (WGA) [24]. The authors compared the performance of ddPCR with CastPCR and concluded that ddPCR was more robust and more sensitive than CastPCR. In this study, the detection of $B R A F$ mutations with ddPCR reached a sensitivity of $0.0005 \%$, which is 200 times greater than that obtained using CastPCR [24].

AS-PCR methods have recently reached sensitivities below $0.01 \%$, which allow their use for rare variant detection [31].

BEAMing technology is sufficiently sensitive $(0.01 \%$, Table 1) to reliably detect mutant ctDNA in the plasma of melanoma patients. For example, $B R A F$ mutations were detectable in $76 \%$ and $81 \%$ of patients with $B R A F$ V600E and V600K mutations among 732 patients enrolled in four recent clinical trials evaluating targeted therapies [34].

The sensitivity of AS-PCR is generally not below $0.1 \%$ $[3,9,19,36]$. However, the recent development of a novel, rapid, and inexpensive AS-PCR assay reached a sensitivity of $0.005 \%$ due to the use of a PNA designed to inhibit the amplification of the wild-type allele [18].

The technique of ddPCR is a robust method that can detect and quantify very small amounts of ctDNA without the need of a calibration curve [28]. It is indeed more precise for the detection of rare genetic variants and is less sensitive to inhibitors than quantitative RT-PCR $[39,40]$. In a recent study, the ddPCR approach reliably distinguished mutant from wild-type alleles with no false positives [29]. BRAF ctDNA was never detected in a healthy patient cohort, yielding a clinical specificity of $100 \%$. Using the same approach, the NRAS ctDNA test showed a specificity higher than $75 \%$ [30].

Most of the technologies presented in this review are generally used to detect and quantify known alterations. Despite being useful for longitudinal monitoring, they do not allow the discovery of other mutations, either the other innumerable mutations contained in the tumor or the de 
TABLE 1: Overview of techniques used for detection and quantification of plasma ctDNA for melanoma patients. PCR: polymerase chain reaction; AS-PCR: allele-specific PCR; ARMS: amplification refractory mutation system allele-specific PCR; MS-PCR: mutant-specific PCR with fluorescent detection; CastPCR: competitive allele-specific PCR; CTCs: circulating tumor cells; Bi-PAP: mutation-specific bidirectional pyrophosphorolysis-activated polymerization; ddPCR: droplet digital PCR; BEAMing: beads, emulsification, amplification, and magnetics; NGS: next generation sequencing; WES: whole exome sequencing.

\begin{tabular}{|c|c|c|c|}
\hline Method & Gene (mutation) & $\begin{array}{l}\text { Analytical sensitivity ( } \% \text { of } \\
\text { mutated copies) }\end{array}$ & References \\
\hline $\begin{array}{l}\text { Quantitative real-time } \\
\text { clamp reverse transcription } \\
\text { PCR }\end{array}$ & $B R A F(\mathrm{p} . \mathrm{V} 600 \mathrm{E})$ & $0.001 \%$ & {$[17]$} \\
\hline AS-PCR or ARMS & $B R A F(\mathrm{p} . \mathrm{V} 600 \mathrm{E} / \mathrm{K} / \mathrm{D})$ & $0.1 \%$ & {$[3]$} \\
\hline AS-PCR or ARMS & $B R A F(\mathrm{p} . \mathrm{V} 600 \mathrm{E})$ & $0.3 \%$ & [18] \\
\hline AS-PCR or ARMS & $B R A F(\mathrm{p} . \mathrm{V} 600 \mathrm{E})$ & $0.25 \%$ & [9] \\
\hline AS-PCR or ARMS & $B R A F(\mathrm{p} . \mathrm{V} 600 \mathrm{E})$ & $2.0 \%$ & [19] \\
\hline ARMS & $\begin{array}{l}\text { BRAF (p.V600E) } \\
B R A F(\mathrm{p} . \mathrm{V} 600 \mathrm{D}) \\
B R A F(\mathrm{p} . \mathrm{V} 600 \mathrm{~K}) \\
B R A F(\mathrm{p} . \mathrm{V} 600 \mathrm{R})\end{array}$ & $\begin{array}{l}1.82 \% \\
3.19 \% \\
4.34 \% \\
4.85 \% \\
\end{array}$ & {$[20]$} \\
\hline MS-PCR & $B R A F(\mathrm{p} . \mathrm{V} 600 \mathrm{E})$ & $0.01 \%$ & {$[21]$} \\
\hline $\begin{array}{l}\text { RT-PCR + restriction } \\
\text { enzyme digestion }\end{array}$ & $B R A F(\mathrm{p} . \mathrm{V} 600 \mathrm{E})$ & $0.1 \%$ & {$[22,23]$} \\
\hline ddPCR on DNA from CTC & $\begin{array}{l}B R A F(\mathrm{p} . \mathrm{V} 600 \mathrm{E}) \\
B R A F(\mathrm{p} . \mathrm{V} 600 \mathrm{~K})\end{array}$ & $\begin{array}{c}0.0005 \% \text { after WGA } \\
\text { enrichment }\end{array}$ & {$[24]$} \\
\hline $\begin{array}{l}\text { CastPCR on DNA from } \\
\text { CTC }\end{array}$ & $\begin{array}{l}B R A F(\mathrm{p} . \mathrm{V} 600 \mathrm{E}) \\
B R A F(\mathrm{p} . \mathrm{V} 600 \mathrm{~K})\end{array}$ & $\begin{array}{c}0.1 \% \text { after WGA } \\
\text { enrichment }\end{array}$ & {$[24]$} \\
\hline CastPCR & $B R A F(\mathrm{p} . \mathrm{V} 600 \mathrm{E})$ & $0.5 \%$ & {$[25]$} \\
\hline Bi-PAP & $\begin{array}{l}\text { GNAQ }(\text { c.626A }>\mathrm{T}) \\
\text { GNAQ }(\mathrm{c} .626 \mathrm{~A}>\mathrm{C}) \\
\text { GNA11 }(\mathrm{c} .626 \mathrm{~A}>\mathrm{T})\end{array}$ & $\sim 0.05 \%$ & {$[26,27]$} \\
\hline ddPCR & $B R A F(\mathrm{p} . \mathrm{V} 600 \mathrm{E})$ & $0.005 \%$ & {$[28]$} \\
\hline ddPCR & $\begin{array}{l}B R A F(\mathrm{p} . \mathrm{V} 600 \mathrm{E}) \\
B R A F(\mathrm{p} . \mathrm{V} 600 \mathrm{~K}) \\
N R A S(\mathrm{p} . \mathrm{Q} 61 \mathrm{H})\end{array}$ & $0.01 \%$ & {$[29]$} \\
\hline ddPCR & $\begin{array}{l}B R A F(\mathrm{p} . \mathrm{V} 600 \mathrm{E}) \\
B R A F(\mathrm{p} . \mathrm{V} 600 \mathrm{~K}) \\
\text { NRAS (p.Q61K) } \\
\text { NRAS (p.Q61R) }\end{array}$ & $0.01 \%$ & {$[30]$} \\
\hline AS-PCR or ARMS & $B R A F(\mathrm{p} . \mathrm{V} 600 \mathrm{E})$ & $0.005 \%$ & {$[18]$} \\
\hline AS-PCR & $B R A F(\mathrm{p} . \mathrm{V} 600 \mathrm{E} / \mathrm{E} 2 / \mathrm{D} / \mathrm{K} / \mathrm{R} / \mathrm{M})$ & $0.01 \%$ & {$[31]$} \\
\hline BEAMing technology & $\begin{array}{l}B R A F(\mathrm{p} . \mathrm{V} 600 \mathrm{E}) \\
B R A F(\mathrm{p} . \mathrm{V} 600 \mathrm{~K})\end{array}$ & & {$[32]$} \\
\hline BEAMing technology & $\begin{array}{l}\text { BRAF (p.V600E) } \\
\text { NRAS (p.Q61K) } \\
\text { NRAS (p.Q61R) }\end{array}$ & $<0.01 \%$ & {$[33]$} \\
\hline BEAMing technology & $\begin{array}{l}B R A F(\mathrm{p} . V 600 \mathrm{E}) \\
B R A F(\mathrm{p} . V 600 \mathrm{~K})\end{array}$ & $0.01 \%$ & {$[34]$} \\
\hline PCR+NGS & TERT promoter & $<0.1 \%$ & {$[33]$} \\
\hline NGS (WES) & Exome & & [35] \\
\hline
\end{tabular}

novo mutations occurring during the acquisition of resistance mechanisms. From this perspective, sequencing of ctDNA via NGS-based technologies is a reliable strategy that has already succeeded in identifying novel alterations at a frequency as low as one mutant copy in several thousand wild-type copies $[41,42]$.
Accordingly, digital PCR and NGS technologies are considered to be relevant and complementary diagnostic tools for quantifying rare variants in the blood of cancer patients $[40,43]$.

Plasma is a better source of ctDNA than serum [19], especially because of the large amounts of wild-type DNA 
released by white cells lysis during clotting. However, discordant studies have shown that higher levels of ctDNA could be recovered from paired serum samples [9]. There is general agreement concerning the use of either dedicated blood collection tubes that prevent plasma cell-free DNA contamination by cellular DNA $[44,45]$ or conventional EDTA-containing tubes coupled with a stringent protocol for the separation of the plasma from blood cells by two consecutive centrifugations within 2-3 hours after the blood draw [46].

Analytical sensitivity (Table 1) refers to the estimated fraction of mutated copies that can be detected within the high number of wild-type alleles present in the plasma. The sensitivity of a given method is a percentage that can vary depending on the allele of interest, the technology used, or the pathophysiological state of the patient. For plasma ctDNA quantitation, the use of very sensitive methods is particularly necessary for quantifying the presence of extremely small fractions of mutant alleles $(<1.0 \%)$. We believe that comparing the absolute sensitivities obtained from various studies aiming to quantify ctDNA is not informative. The diverse experimental protocols used in these studies introduce bias, preventing their comparison. The analysis of ctDNA coming from different sources (plasma, serum, or CTCs), with or without rapid processing after blood collection, obtained using different extraction methods, followed by whole genome amplification or not, and sequenced/quantified using different technologies, impedes any attempt to select the best approach for ctDNA quantification based on claimed (or measured) analytical sensitivities [47]. Importantly, most plasma or serum ctDNA is not derived from CTCs [7].

\section{Clinical Applications for ctDNA in Melanoma}

The recent advances resulting in improved sensitivity and specificity of ctDNA analysis have been shown to be useful in a wide variety of malignancies for several clinical applications, including early cancer detection, assessment of the molecular heterogeneity of overall disease, the monitoring of tumor dynamics, identification of genetic determinants for targeted therapy, evaluation of early treatment responses, monitoring of minimal residual disease, and assessment of the evolution of resistance in real time [38]. Several of these applications have been tested on melanoma patients and the results all support the serious need for routine serial ctDNA monitoring to improve the clinical management of these patients (Table 2).

3.1. Assessment of Molecular Heterogeneity. The assessment of genetic heterogeneity is a current technical challenge of major clinical importance. As an example, the mutational status of $B R A F$ was found to be different between different sites of the primary tumor (intratumor heterogeneity), between the primary tumor and metastases, and between several metastases from the same patient $[21,48]$. Biopsy and subsequent sequencing can miss detecting a mutation because of the site from which it was taken. A potential strategy to overcome such a bias in routine sequencing is to sequence plasma
ctDNA. Indeed, ctDNA is a valid source for theoretically finding all mutations present in all tumor sites of a given patient [38].

3.2. Prognostic Value. The highest levels of circulating BRAF $\mathrm{V} 600 \mathrm{E}$ were found in more advanced stages of disease [17]. This finding was in accordance with results of another seminal study, dedicated to quantifying circulating $B R A F$ mutations in the blood of melanoma patients, which showed that detectable $B R A F$ levels were only found in advanced stage III/IV disease [9]. The authors of this study postulated that this blood biomarker would not represent an interesting early-stage marker for melanoma patients, despite the fact that BRAF mutations occur early in melanomagenesis [49].

The prognostic significance of ctDNA quantification in melanoma has more recently been clearly demonstrated. Patients with OS of more than two years had significantly fewer copies of mutant BRAF V600E per $\mathrm{mL}$ of plasma than patients with OS of less than two years [28]. The prognostic value of ctDNA levels was also demonstrated in a phase II cohort of melanoma patients which included histologically confirmed patients with $B R A F$ mutant stage IV melanoma [32]. Increasing amounts of circulating $B R A F$ $\mathrm{V} 600 \mathrm{E}$ were associated with a reduced overall response rate and shorter progression-free survival (PFS). Elevated ctDNA levels were also significantly associated with both shorter PFS and lower OS in uveal melanoma [26]. In addition, the absence of detectable ctDNA prior to treatment (baseline) within a large cohort of melanoma patients with $B R A F$ mutations was an independent predictor of better disease outcome. Patients negative for BRAF ctDNA had higher response rates to dabrafenib and trametinib, longer PFS, and higher OS than patients for whom $B R A F$ mutations were detected in the plasma [34]. Longer PFS also correlated with undetectable ctDNA levels after treatment initiation in an independent 36-patient cohort receiving dabrafenib and trametinib [31]. In a 48-patient cohort treated by MAPK inhibitors or immunotherapies, low baseline ctDNA was a good predictor of response to treatment and longer PFS [30]. More recently, the presence of detectable levels of ctDNA at baseline strongly correlated with longer OS [20]. All these studies suggest that rising or elevated ctDNA levels may predict poorer clinical outcome.

\subsection{Identification of Genetic Determinants for Targeted Ther-} apy. A phase II study of a large cohort of BRAF mutant melanoma patients failed to demonstrate the prognostic value of ctDNA detection in advanced melanoma. However, the authors stressed that there was a strong rationale for using blood testing to identify whether there are any genetic determinants for associated targeted therapy [3]. The definitive evidence of the benefit of using plasma rather than serum for ctDNA studies was provided in a large cohort of melanoma patients [19]. In this study, the authors proposed that $B R A F$ V600 mutation testing from ctDNA should be considered as a first screening test for positively selecting patients with $B R A F$ mutations eligible for targeted therapy. This blood-based mutation screening approach could significantly shorten the 
TABLE 2: Applications of ctDNA quantification and monitoring for melanoma patients. PBL: peripheral blood lymphocytes.

\begin{tabular}{|c|c|c|c|}
\hline Gene & Sample & Application & References \\
\hline$B R A F$ & Serum & $\begin{array}{l}\text { (i) Tumor response monitoring } \\
\text { (ii) Prognostic value }\end{array}$ & [17] \\
\hline$B R A F$ & Serum/plasma & Advanced stage IV monitoring & [9] \\
\hline$B R A F$ & Serum & $\begin{array}{l}\text { Identification of genetic determinants for targeted } \\
\text { therapy }\end{array}$ & {$[3]$} \\
\hline$B R A F$ & Plasma & $\begin{array}{l}\text { Identification of genetic determinants for targeted } \\
\text { therapy }\end{array}$ & {$[21]$} \\
\hline$B R A F$ & Serum/plasma & $\begin{array}{l}\text { Identification of genetic determinants for targeted } \\
\text { therapy }\end{array}$ & {$[19]$} \\
\hline$B R A F$ & Plasma & $\begin{array}{l}\text { Identification of genetic determinants for targeted } \\
\text { therapy }\end{array}$ & {$[36]$} \\
\hline$B R A F$ & PBL & $\begin{array}{l}\text { (i) Identification of genetic determinants for targeted } \\
\text { therapy } \\
\text { (ii) Evaluation of early treatment response } \\
\text { (iii) Monitoring of minimal residual disease }\end{array}$ & {$[22]$} \\
\hline $\begin{array}{l}\text { GNAQ } \\
\text { GNA11 }\end{array}$ & Plasma & Prognostic value & {$[26]$} \\
\hline$B R A F$ & Plasma & $\begin{array}{l}\text { (i) Identification of genetic determinants for targeted } \\
\text { therapy } \\
\text { (ii) Monitoring of tumor dynamics } \\
\text { (iii) Prognostic value }\end{array}$ & {$[28]$} \\
\hline $\begin{array}{l}\text { BRAF } \\
\text { NRAS } \\
\text { TERT } \\
\text { Promoter }\end{array}$ & Plasma & $\begin{array}{l}\text { Evaluation of early treatment response to } \\
\text { immunotherapy }\end{array}$ & {$[33]$} \\
\hline$B R A F$ & Plasma/serum & $\begin{array}{l}\text { (i) Prognostic value } \\
\text { (ii) Monitoring of tumor dynamics } \\
\text { (iii) Evaluation of early treatment response } \\
\text { (iv) Monitoring of minimal residual disease }\end{array}$ & {$[18]$} \\
\hline$B R A F$ & Plasma & Prognostic value & {$[32]$} \\
\hline $\begin{array}{l}B R A F \\
N R A S\end{array}$ & Plasma & $\begin{array}{l}\text { (i) Monitoring of tumor dynamics } \\
\text { (ii) Monitoring of minimal residual disease } \\
\text { (iii) Real-time assessment of resistance }\end{array}$ & {$[30]$} \\
\hline$B R A F$ & Plasma & Prognostic value & {$[34]$} \\
\hline$B R A F$ & Plasma & $\begin{array}{l}\text { (i) Prognostic value } \\
\text { (ii) Monitoring of tumor dynamics } \\
\text { (iii) Evaluation of early treatment response } \\
\text { (iv) Real-time assessment of resistance }\end{array}$ & {$[34]$} \\
\hline $\begin{array}{l}B R A F \\
\text { NRAS }\end{array}$ & Plasma & $\begin{array}{l}\text { (i) Prognostic value } \\
\text { (ii) Evaluation of early treatment response } \\
\text { (iii) Real-time assessment of resistance }\end{array}$ & {$[30]$} \\
\hline$B R A F$ & Plasma & $\begin{array}{l}\text { (i) Identification of genetic determinants for targeted } \\
\text { therapy } \\
\text { (ii) Prognostic value }\end{array}$ & {$[20]$} \\
\hline Exome & Plasma & $\begin{array}{l}\text { (i) Evaluation of early treatment response } \\
\text { (ii) Real-time assessment of resistance }\end{array}$ & {$[35]$} \\
\hline
\end{tabular}

turnaround time relative to that required for mutation testing from archived FFPE tumors [19].

3.4. Monitoring of the Disease. The first attempt to monitor ctDNA levels in melanoma patients before and after treatment resulted in a significant positive correlation between the level of remaining detectable serum BRAF V600E and the absence of response to bio/chemotherapy [17]. Another study reported a rapid and marked decrease in $B R A F$ V600E blood levels following the initiation of treatment, whether it was surgical or based on targeted anti-BRAF and/or antiMEK therapies. The decrease correlated with the response to treatment, shown by imaging [22].

The longitudinal variations of ctDNA levels during the treatment of melanoma are logarithmic [28], reinforcing its potential role as a relevant surrogate marker for monitoring 
efficacy (decrease and/or minimal residual disease) and recurrence (increase after nadir) of melanoma therapy. The only blood TM routinely incorporated in the management of melanoma is LDH, but it is neither sensitive nor specific and is considered to be an unreliable marker for monitoring treatment response [29]. A small case series demonstrated that ddPCR-based ctDNA quantification allowed the monitoring of treatment responses or the emergence of tumor resistance more consistently and informatively than $\mathrm{LDH}$ [29]. The superiority of measuring ctDNA over that of $\mathrm{LDH}$ levels was demonstrated in three other patients for whom ctDNA levels more accurately reflected the evolution of disease than those of $\mathrm{LDH}$, as the latter tends to increase following immunotherapy [35]. In contrast, two other studies reported a positive correlation between ctDNA and $\mathrm{LDH}$ levels $[20,30]$.

Changes in ctDNA levels that occurred in patients receiving immune checkpoint blocking agents were predictive of treatment efficacy [33]. In this study, the authors also postulated that (1) decreases in ctDNA levels could help to identify patients who are responding to treatment, (2) increases in ctDNA levels might indicate tumor progression or tumor lysis preceding regression, (3) rising ctDNA levels after a long period off treatment might reflect tumor recurrence, and (4) ctDNA could be used as a marker of minimal residual disease after surgical resection. The monitoring of metastatic melanoma patients receiving targeted therapies also suggested that ctDNA levels dramatically decline during the first weeks of therapy, sometimes decreasing to undetectable levels, which is also associated with response to therapy [30]. An increase of ctDNA levels was never found in patients with an ongoing response but was found in 19/27 patients with progressive disease under targeted therapy, thus resulting in a sensitivity of $70 \%$ and a specificity of $100 \%$ [31].

The interest of such a longitudinal follow-up is that the recurrence of tumor burden was apparent by ctDNA analysis when radiological analysis still classified the tumor as stable [30]. That biological recurrence precedes radiological signs of recurrence by several weeks is well established $[50,51]$.

3.5. Resistance to Treatment. The assessment of tumor resistance to a given treatment can be determined by ctDNA studies in a dual manner. The first option consists of detecting increased levels of ctDNA carrying the original mutation after a prolonged period of undetectable or low values. $B R A F$ mutations are usually conserved when recurrence/resistance to targeted therapies occurs and concentrations of plasma ctDNA generally decrease after treatment initiation [30]. Therefore, if progressive disease occurs after a period of tumor response, it is likely that an increase of ctDNA levels with the same primary $B R A F$ mutation as that of the relapsing tumor cells will be detected. The second option consists of detecting resistance mutations. For melanoma treated with BRAF inhibitors, resistance generally occurs within the first year and usually involves reactivation of the MAPK pathway by the acquisition of secondary NRAS or MEK mutations or alternative splicing or amplification of the $B R A F$ gene itself [52]. Tracking such secondary mutations is an approach that has already demonstrated its utility in the routine clinical monitoring for the early recurrence of lung cancers [53]. For melanoma patients, ctDNA monitoring of seven patients with progressive disease after a prior response to anti-BRAF treatments allowed the identification of secondary resistance mutations of the NRAS gene for three of them [30]. Similarly, a retrospective analysis of ctDNA from patients with tumor resistance was also performed by whole exome sequencing (WES) [35] and identified secondary NRAS mutations. A specific 10-gene panel was further developed and successfully used for the assessment in plasma of secondary mutations known to mediate resistance to BRAF and MEK inhibitors [35].

\section{General Conclusion}

Liquid biopsies are a promising area of investigation for improving the clinical monitoring of patients with solid malignancies [7] and ctDNA has been demonstrated for several applications to be a diagnostic, prognostic, and predictive biomarker for several malignancies [47]. The recent progress in the analytical sensitivity of digital genomic technologies allows the investigation of very rare mutant variants of ctDNA, representing an indisputable advantage over most strategies requiring CTC isolation [7]. The routine sequencing of BRAF (exon 15), NRAS (exon 2), and KIT (exons 9, 11,13 , and 17) for clinical purposes, such as molecular-based targeted therapy, suggests that tailored monitoring of the kinetics of plasma ctDNA could be used in the near future for melanoma patients for whom a mutation is detected.

Indeed, ctDNA can also harbor epigenetic modifications, such as methylation that has already been identified as a relevant prognostic factor for melanoma patients [54, 55]. Digital PCR and NGS strategies appear to be two of the most versatile technologies to study rare variants, such as mutations for cancer or SNPs for pharmacogenetics, as well as methylation [56].

Given the heterogeneity of tumor mutations between primary and metastatic sites, or between several metastatic sites, ctDNA might provide a more relevant representation of the mutational status of a patient than a biopsy from a single lesion or site $[3,20,28]$. Liquid biopsies, especially plasma ctDNA, have made the analysis of acquired resistance to anticancer treatments possible [50]. Recent studies showed that the monitoring of plasma ctDNA accurately reflected real-time sampling of multifocal tumor evolution [57]. These findings are fully transposable to melanoma patients, and such monitoring will help clinicians to better understand the molecular evolution of the individual primary and metastatic tumors of their patients. Further studies on large cohorts will be needed to prospectively evaluate the benefit of using serial ctDNA investigations in the monitoring of melanoma patients.

\section{Conflicts of Interest}

The authors have no conflicts of interest to declare. 


\section{Acknowledgments}

This study was funded by "Association Biologie et Cancer," Roche, and Novartis.

\section{References}

[1] A. Ziegler, U. Zangemeister-Wittke, and R. A. Stahel, "Circulating DNA: a new diagnostic gold mine?" Cancer Treatment Reviews, vol. 28, no. 5, pp. 255-271, 2002.

[2] H. Y. Wong and B. H. Park, "Plasma tumor DNA: on your markers, get set, go!," Annals of Translational Medicine, vol. 2, no. 1, article 2, 2014.

[3] R. E. Board, G. Ellison, M. C. M. Orr et al., "Detection of BRAF mutations in the tumour and serum of patients enrolled in the AZD6244 (ARRY-142886) advanced melanoma phase II study," British Journal of Cancer, vol. 101, no. 10, pp. 1724-1730, 2009.

[4] S. J. Dawson, N. Rosenfeld, and C. Caldas, "Circulating tumor DNA to monitor metastatic breast cancer," New England Journal of Medicine, vol. 369, no. 1, pp. 93-94, 2013.

[5] A. Díaz-Lagares, E. Alegre, A. Arroyo et al., "Evaluation of multiple serum markers in advanced melanoma," Tumour Biology, vol. 32, no. 6, pp. 1155-1161, 2011.

[6] M. S. Lawrence, P. Stojanov, P. Polak et al., "Mutational heterogeneity in cancer and the search for new cancer-associated genes," Nature, vol. 499, no. 7457, pp. 214-218, 2013.

[7] C. Bettegowda, M. Sausen, R. J. Leary et al., "Detection of circulating tumor DNA in early- and late-stage human malignancies," Science Translational Medicine, vol. 6, no. 224, Article ID 224ra24, 2014.

[8] E. Hodis, I. R. Watson, G. V. Kryukov et al., "A landscape of driver mutations in melanoma," Cell, vol. 150, no. 2, pp. 251-263, 2012.

[9] M. Daniotti, V. Vallacchi, L. Rivoltini et al., "Detection of mutated BRAFV600E variant in circulating DNA of stage IIIIV melanoma patients," International Journal of Cancer, vol. 120, no. 11, pp. 2439-2444, 2007.

[10] P. T. C. Wan, M. J. Garnett, S. M. Roe et al., "Mechanism of activation of the RAF-ERK signaling pathway by oncogenic mutations of B-RAF," Cell, vol. 116, no. 6, pp. 855-867, 2004.

[11] C. Milagre, N. Dhomen, F. C. Geyer et al., "A mouse model of melanoma driven by oncogenic KRAS," Cancer Research, vol. 70, no. 13, pp. 5549-5557, 2010.

[12] H. L. Vu and A. E. Aplin, "Targeting mutant NRAS signaling pathways in melanoma," Pharmacological Research, vol. 107, pp. 111-116, 2016.

[13] J. A. Jakob, R. L. Bassett Jr., C. S. Ng et al., "NRAS mutation status is an independent prognostic factor in metastatic melanoma," Cancer, vol. 118, no. 16, pp. 4014-4023, 2012.

[14] G. Kang, B. N. Bae, B. S. Sohn, J.-S. Pyo, G. H. Kang, and K.-M. Kim, "Detection of KIT and PDGFRA mutations in the plasma of patients with gastrointestinal stromal tumor," Targeted Oncology, vol. 10, no. 4, pp. 597-601, 2015.

[15] N. Wada, Y. Kurokawa, T. Takahashi et al., "Detecting secondary C-KIT mutations in the peripheral blood of patients with imatinib-resistant gastrointestinal stromal tumor," Oncology, vol. 90, no. 2, pp. 112-117, 2016.

[16] D. Handolias, A. L. Hamilton, R. Salemi et al., "Clinical responses observed with imatinib or sorafenib in melanoma patients expressing mutations in KIT,' British Journal of Cancer, vol. 102, no. 8, pp. 1219-1223, 2010.
[17] M. Shinozaki, S. J. O’Day, M. Kitago et al., "Utility of circulating B-RAF DNA mutation in serum for monitoring melanoma patients receiving biochemotherapy," Clinical Cancer Research, vol. 13, no. 7, pp. 2068-2074, 2007.

[18] M. Gonzalez-Cao, C. Mayo-De-Las-Casas, M. A. Molina-Vila et al., "BRAF mutation analysis in circulating free tumor DNA of melanoma patients treated with BRAF inhibitors," Melanoma Research, vol. 25, no. 6, pp. 486-495, 2015.

[19] K. L. Aung, E. Donald, G. Ellison et al., "Analytical validation of BRAF mutation testing from circulating free DNA using the amplification refractory mutation testing system," Journal of Molecular Diagnostics, vol. 16, no. 3, pp. 343-349, 2014.

[20] A. C. Knol, A. Vallée, G. Herbreteau et al., "Clinical significance of BRAF mutation status in circulating tumor DNA of metastatic melanoma patients at baseline," Experimental Dermatology, vol. 25, no. 10, pp. 783-788, 2016.

[21] M. Yancovitz, A. Litterman, J. Yoon et al., "Intra- and intertumor heterogeneity of $B R A F^{V 600 E}$ mutations in primary and metastatic melanoma," PLoS ONE, vol. 7, no. 1, Article ID e29336, 2012.

[22] D. J. Panka, E. Buchbinder, A. Giobbie-Hurder et al., "Clinical utility of a blood-based $\mathrm{BRAF}^{\mathrm{V} 600 \mathrm{E}}$ mutation assay in melanoma," Molecular Cancer Therapeutics, vol. 13, no. 12, pp. 3210-3218, 2014.

[23] D. J. Panka, R. J. Sullivan, and J. W. Mier, "An inexpensive specific and highly sensitive protocol to detect the BrafV600E mutation in melanoma tumor biopsies and blood," Melanoma Research, vol. 20, no. 5, pp. 401-407, 2010.

[24] A. L. Reid, J. B. Freeman, M. Millward, M. Ziman, and E. S. Gray, "Detection of BRAF-V600E and V600K in melanoma circulating tumour cells by droplet digital PCR," Clinical Biochemistry, vol. 48, no. 15, pp. 999-1002, 2015.

[25] A. Ashida, K. Sakaizawa, A. Mikoshiba, H. Uhara, and R. Okuyama, "Quantitative analysis of the BRAF" ${ }^{\mathrm{V} 600 \mathrm{E}}$ mutation in circulating tumor-derived DNA in melanoma patients using competitive allele-specific TaqMan PCR," International Journal of Clinical Oncology, vol. 21, no. 5, pp. 981-988, 2016.

[26] F.-C. Bidard, J. Madic, P. Mariani et al., "Detection rate and prognostic value of circulating tumor cells and circulating tumor DNA in metastatic uveal melanoma," International Journal of Cancer, vol. 134, no. 5, pp. 1207-1213, 2014.

[27] J. Madic, S. Piperno-Neumann, V. Servois et al., "Pyrophosphorolysis-activated polymerization detects circulating tumor DNA in metastatic uveal melanoma," Clinical Cancer Research, vol. 18, no. 14, pp. 3934-3941, 2012.

[28] M. F. Sanmamed, S. Fernandez-Landazuri, C. Rodriguez et al., "Quantitative cell-free circulating BRAFV600E mutation analysis by use of droplet digital PCR in the follow-up of patients with melanoma being treated with BRAF inhibitors," Clinical Chemistry, vol. 61, no. 1, pp. 297-304, 2015.

[29] S. Chang-Hao Tsao, J. Weiss, C. Hudson et al., "Monitoring response to therapy in melanoma by quantifying circulating tumour DNA with droplet digital PCR for BRAF and NRAS mutations," Scientific Reports, vol. 5, article 11198, 2015.

[30] E. S. Gray, H. Rizos, A. L. Reid et al., "Circulating tumor DNA to monitor treatment response and detect acquired resistance in patients with metastatic melanoma," Oncotarget, vol. 6, no. 39, pp. 42008-42018, 2015.

[31] M. Schreuer, G. Meersseman, S. Van Den Herrewegen et al., "Quantitative assessment of BRAF V600 mutant circulating cell-free tumor DNA as a tool for therapeutic monitoring 
in metastatic melanoma patients treated with BRAF/MEK inhibitors," Journal of Translational Medicine, vol. 14, article 95, 2016.

[32] P. A. Ascierto, D. Minor, A. Ribas et al., "Phase II trial (BREAK2) of the BRAF inhibitor dabrafenib (GSK2118436) in patients with metastatic melanoma," Journal of Clinical Oncology, vol. 31, no. 26, pp. 3205-3211, 2013.

[33] E. J. Lipson, V. E. Velculescu, T. S. Pritchard et al., "Circulating tumor DNA analysis as a real-time method for monitoring tumor burden in melanoma patients undergoing treatment with immune checkpoint blockade," Journal for ImmunoTherapy of Cancer, vol. 2, no. 1, article 42, 2014.

[34] A. Santiago-Walker, R. Gagnon, J. Mazumdar et al., "Correlation of BRAF mutation status in circulating-free DNA and tumor and association with clinical outcome across four BRAFi and MEKi clinical trials," Clinical Cancer Research, vol. 22, no. 3, pp. 567-574, 2016.

[35] M. R. Girotti, G. Gremel, R. Lee et al., "Application of sequencing, liquid biopsies, and patient-derived xenografts for personalized medicine in melanoma," Cancer Discovery, vol. 6, no. 3, pp. 286-299, 2016.

[36] P. Pinzani, F. Salvianti, R. Cascella et al., "Allele specific Taqmanbased real-time PCR assay to quantify circulating BRAFV600E mutated DNA in plasma of melanoma patients," Clinica Chimica Acta, vol. 411, no. 17-18, pp. 1319-1324, 2010.

[37] M. Fleischhacker and B. Schmidt, "Circulating nucleic acids (CNAs) and cancer-a survey," Biochimica et Biophysica Acta, vol. 1775, no. 1, pp. 181-232, 2007.

[38] L. A. Diaz Jr. and A. Bardelli, "Liquid biopsies: genotyping circulating tumor DNA," Journal of Clinical Oncology, vol. 32, no. 6, pp. 579-586, 2014.

[39] T. C. Dingle, R. H. Sedlak, L. Cook, and K. R. Jerome, "Tolerance of droplet-digital PCR vs real-time quantitative PCR to inhibitory substances," Clinical Chemistry, vol. 59, no. 11, pp. 1670-1672, 2013.

[40] J. F. Huggett, S. Cowen, and C. A. Foy, "Considerations for digital PCR as an accurate molecular diagnostic tool," Clinical Chemistry, vol. 61, no. 1, pp. 79-88, 2015.

[41] T. Forshew, M. Murtaza, C. Parkinson et al., "Noninvasive identification and monitoring of cancer mutations by targeted deep sequencing of plasma DNA," Science Translational Medicine, vol. 4, no. 136, Article ID 136ra68, 2012.

[42] I. Kinde, J. Wu, N. Papadopoulos, K. W. Kinzler, and B. Vogelstein, "Detection and quantification of rare mutations with massively parallel sequencing," Proceedings of the National Academy of Sciences of the United States of America, vol. 108, no. 23, pp. 9530-9535, 2011.

[43] D. A. Haber and V. E. Velculescu, "Blood-based analyses of cancer: circulating tumor cells and circulating tumor DNA," Cancer Discovery, vol. 4, no. 6, pp. 650-661, 2014.

[44] S. E. Norton, J. M. Lechner, T. Williams, and M. R. Fernando, "A stabilizing reagent prevents cell-free DNA contamination by cellular DNA in plasma during blood sample storage and shipping as determined by digital PCR," Clinical Biochemistry, vol. 46, no. 15, pp. 1561-1565, 2013.

[45] P. V. Toro, B. Erlanger, J. A. Beaver et al., "Comparison of cell stabilizing blood collection tubes for circulating plasma tumor DNA," Clinical Biochemistry, vol. 48, no. 15, pp. 993-998, 2015.

[46] X. Xue, M. D. Teare, I. Holen, Y. M. Zhu, and P. J. Woll, "Optimizing the yield and utility of circulating cell-free DNA from plasma and serum," Clinica Chimica Acta, vol. 404, no. 2, pp. 100-104, 2009.
[47] E. Heitzer, P. Ulz, and J. B. Geigl, "Circulating tumor DNA as a liquid biopsy for cancer," Clinical Chemistry, vol. 61, no. 1, pp. 112-123, 2015.

[48] J. Lin, Y. Goto, H. Murata et al., "Polyclonality of BRAF mutations in primary melanoma and the selection of mutant alleles during progression," British Journal of Cancer, vol. 104, no. 3, pp. 464-468, 2011.

[49] K. Omholt, A. Platz, L. Kanter, U. Ringborg, and J. Hansson, "NRAS and BRAF mutations arise early during melanoma pathogenesis and are preserved throughout tumor progression," Clinical Cancer Research, vol. 9, no. 17, pp. 6483-6488, 2003.

[50] M. Murtaza, S.-J. Dawson, D. W. Y. Tsui et al., "Non-invasive analysis of acquired resistance to cancer therapy by sequencing of plasma DNA," Nature, vol. 497, no. 7447, pp. 108-112, 2013.

[51] A. M. Newman, S. V. Bratman, J. To et al., "An ultrasensitive method for quantitating circulating tumor DNA with broad patient coverage," Nature Medicine, vol. 20, no. 5, pp. 548-554, 2014.

[52] J. Charles, C. Martel, F. De Fraipont, M.-T. Leccia, C. Robert, and B. Busser, "Mechanisms of resistance to anti-BRAF treatments," Annales de Dermatologie et de Venereologie, vol. 141, no. 11, pp. 671-681, 2014.

[53] G. R. Oxnard, C. P. Paweletz, Y. Kuang et al., "Noninvasive detection of response and resistance in EGFR-mutant lung cancer using quantitative next-generation genotyping of cellfree plasma DNA," Clinical Cancer Research, vol. 20, no. 6, pp. 1698-1705, 2014.

[54] T. Mori, S. J. O’Day, N. Umetani et al., "Predictive utility of circulating methylated DNA in serum of melanoma patients receiving biochemotherapy," Journal of Clinical Oncology, vol. 23, no. 36, pp. 9351-9358, 2005.

[55] K. Koyanagi, T. Mori, S. J. O’Day, S. R. Martinez, H.-J. Wang, and D. S. B. Hoon, "Association of circulating tumor cells with serum tumor-related methylated DNA in peripheral blood of melanoma patients," Cancer Research, vol. 66, no. 12, pp. 61116117, 2006.

[56] E. Day, P. H. Dear, and F. McCaughan, "Digital PCR strategies in the development and analysis of molecular biomarkers for personalized medicine," Methods, vol. 59, no. 1, pp. 101-107, 2013.

[57] M. Murtaza, S.-J. Dawson, K. Pogrebniak et al., "Multifocal clonal evolution characterized using circulating tumour DNA in a case of metastatic breast cancer," Nature Communications, vol. 6, article 8760, 2015. 


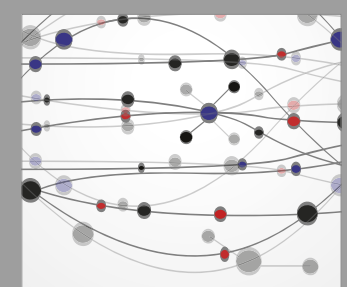

The Scientific World Journal
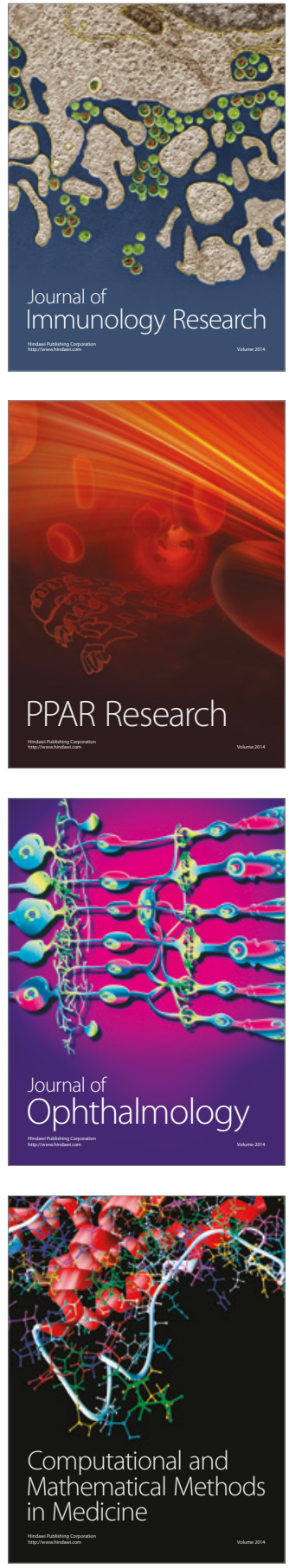

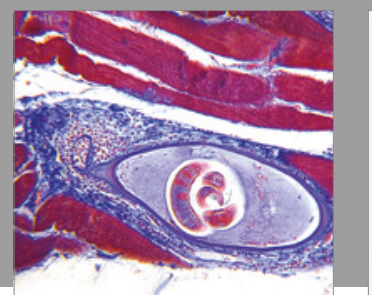

Gastroenterology Research and Practice
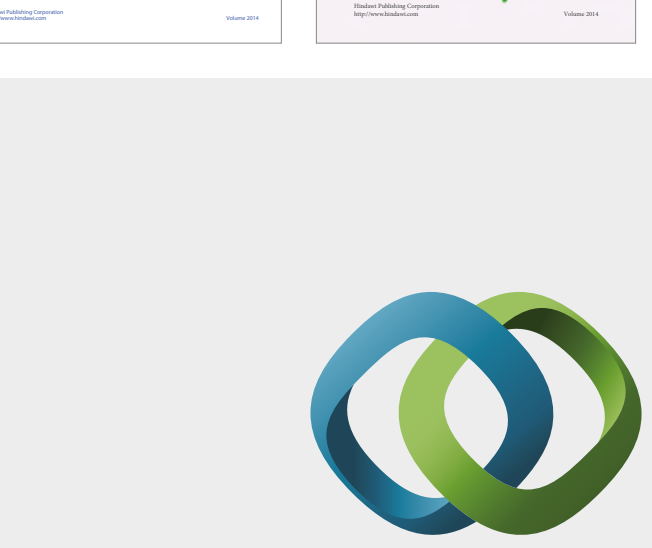

\section{Hindawi}

Submit your manuscripts at

https://www.hindawi.com
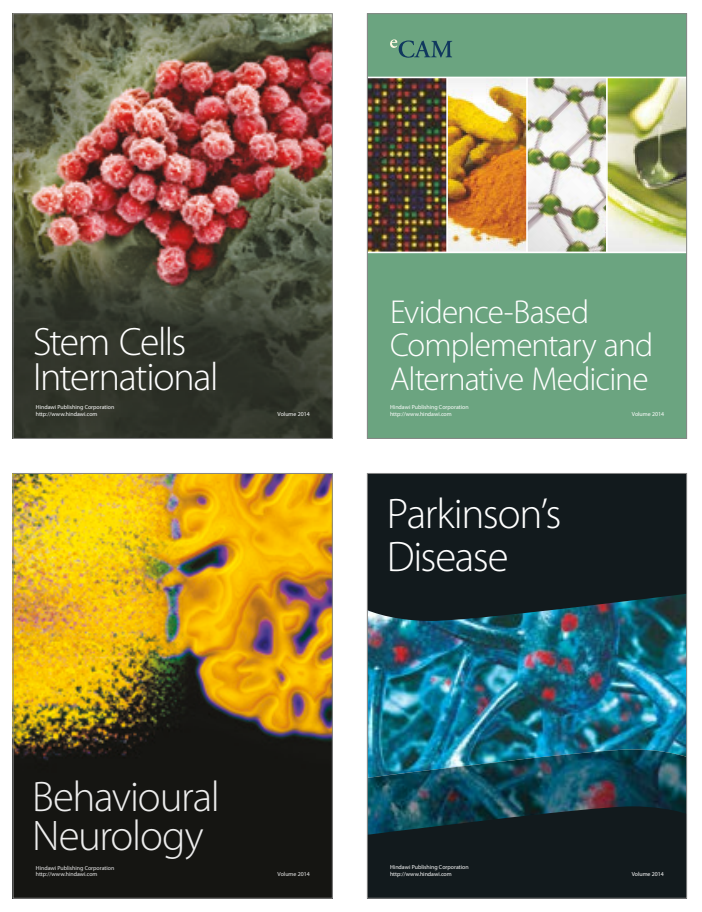
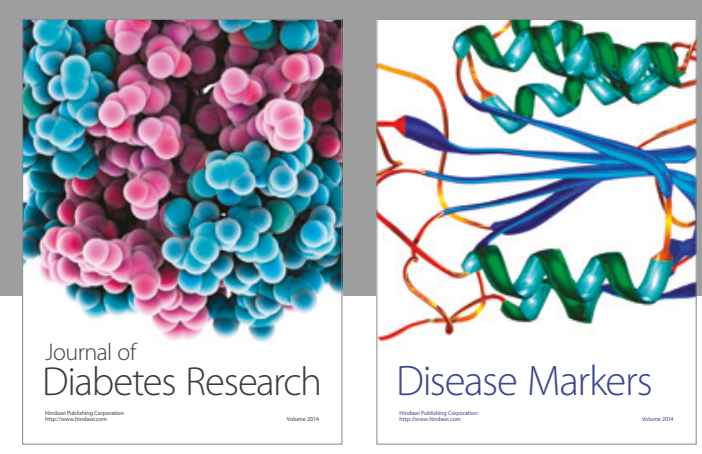

Disease Markers
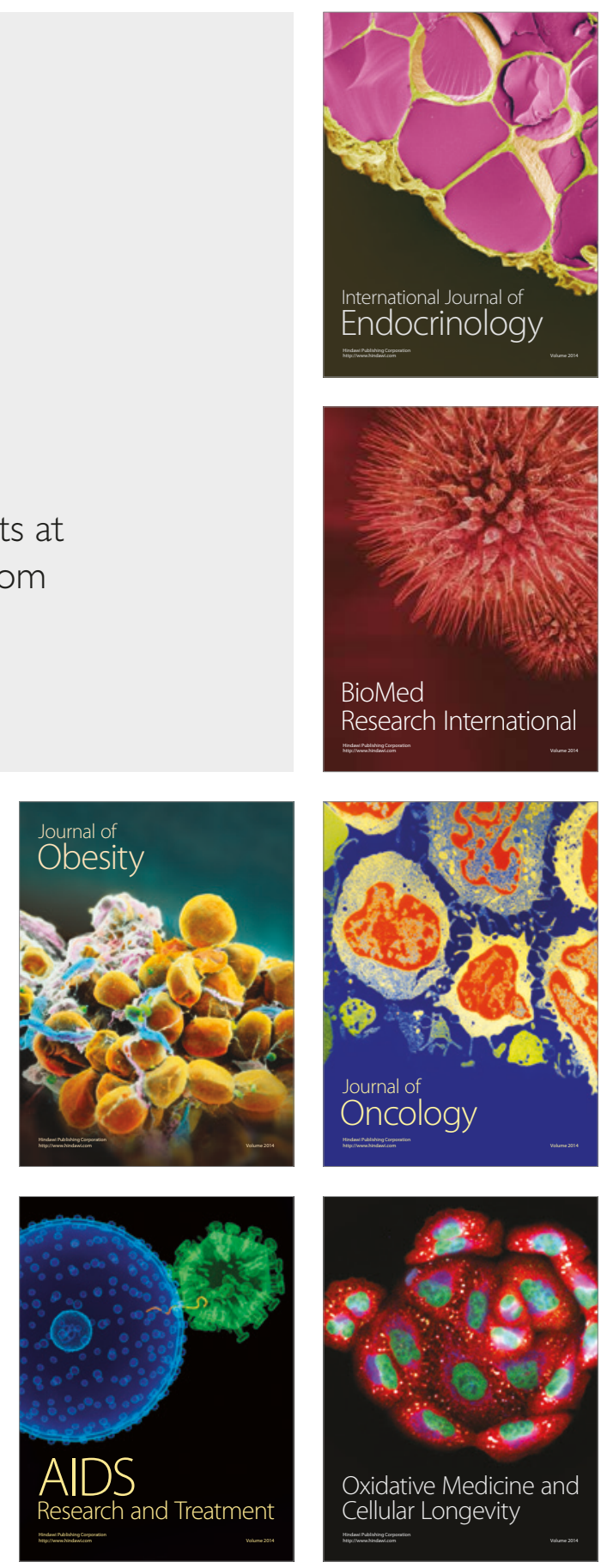